\title{
The Effect of Educational Program on Self-Management of Patients with Epilepsy: A Randomized Clinical Trial Study
}

\author{
Narges Bahiraei ${ }^{1}$, Maryam Dehghani ${ }^{2}$, Alice Khachian ${ }^{3 *}$ \\ 1. Department of Medical Surgical Nursing, School of Nahavand Paramedical, Hamadan University of Medical Sciences, Hamadan, Iran \\ 2. Department of Pediatrics Nursing, School of Nahavand Paramedical, Hamadan University of Medical Sciences, Hamadan, Iran \\ 3. Department of Medical-Surgical Nursing, Assistant Professor, Faculty of Nursing and Midwifery, School of Nursing and Midwifery, Iran \\ University of Medical Sciences, Tehran, Iran
}

\begin{tabular}{|c|c|}
\hline Article Info & ABSTRACT \\
\hline $\begin{array}{l}\text { Received: 2018/12/25; } \\
\text { Accepted: 2019/03/04; } \\
\text { Published Online: 2019/03/27 }\end{array}$ & $\begin{array}{l}\text { Introduction: Patients with epilepsy need to learn to identify seizure-inducing } \\
\text { factors in order to adapt to seizure activity and disease management and taking } \\
\text { precautionary measures to minimize seizure damage. The aim of this study was to } \\
\text { determine the effect of an educational program on self-management empowerment of } \\
\text { patients with epilepsy. }\end{array}$ \\
\hline
\end{tabular}

Methods: This quasi-experimental study was conducted on 90 patients with epilepsy referring to Iranian Epilepsy Association. The study used convenient sampling and samples were randomly divided into two groups: control $(n=45)$ and experimental group $(n=45)$. The control group received routine training and the experimental group in addition to routine training, participated in three educational sessions held by the researcher in Epilepsy Association. All the patients completed a self-management questionnaire before and six weeks after the intervention. The data were analyzed by SPSS 22 using Kolmogorov-Smirnov, independent paired t-test and descriptive statistics.

Results: The findings showed that there were no significant differences between the two groups in terms of demographic characteristics $(P>0.05)$. Before implementing educational program, the two groups showed no significant difference in medication, knowledge, information, safety and seizure scores $(P>0.05)$. After implementing educational program the two groups showed significant difference in the scores $(P<0.01)$, which is indicative of the increased scores in experimental group.

Conclusion: The results of the study indicated that the education has been effective in information, seizure control and safety of people with epilepsy. Implementation of such programs in order to better accommodate people with epilepsy with their illness is recommended.

Keywords: Education, Epilepsy, Self-management

Corresponding Information:

Alice Khachian, Department of Medical-Surgical Nursing, Faculty of Nursing and Midwifery, School of Nursing and Midwifery, Iran University of Medical Sciences, Tehran, Iran. Email: alicekhachian@hotmail.com

Copyright @ 2019, This is an original open-access article distributed under the terms of the Creative Commons Attribution-noncommercial 4.0 International License which permits copy and redistribution of the material just in noncommercial usages with proper citation.

\section{How to Cite This Article:}

Bahiraei N, Dehghani M, Khachian A. The Effect of Educational Program on Self-Management of Patients with Epilepsy: A Randomized Clinical Trial Study. Avicenna J Nurs Midwifery care. 2019; 27 (5) : $361-369$ 


\section{تأثير بهكاركيرى برناملُ آموزشى بر خودمديريتى افراد مبتلا به صرع: مطالعلُ كار آزمايى بالينى}

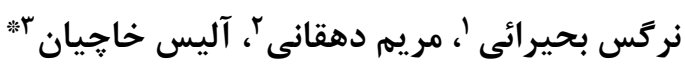

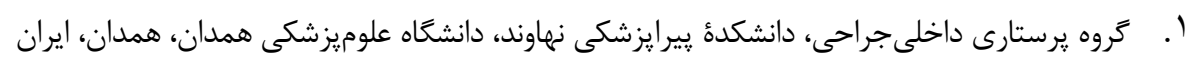

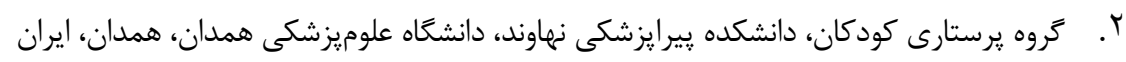

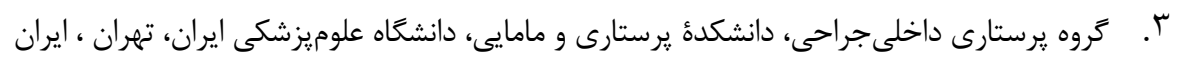

\begin{tabular}{|c|c|}
\hline "جكيده & اطلاعات مقاله \\
\hline 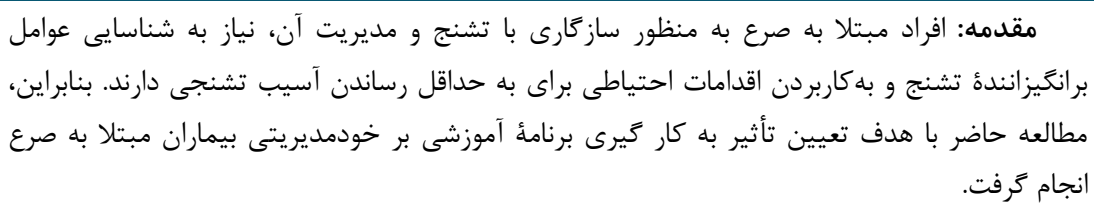 & 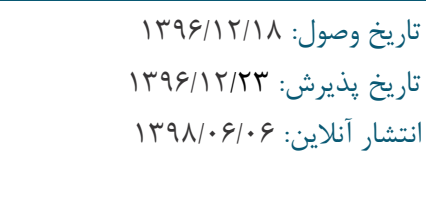 \\
\hline 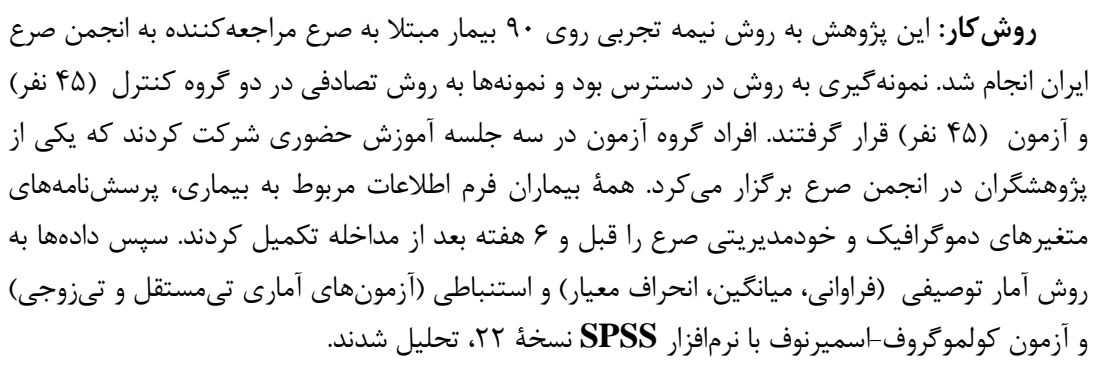 & 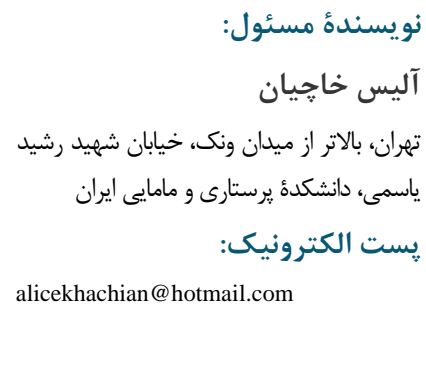 \\
\hline 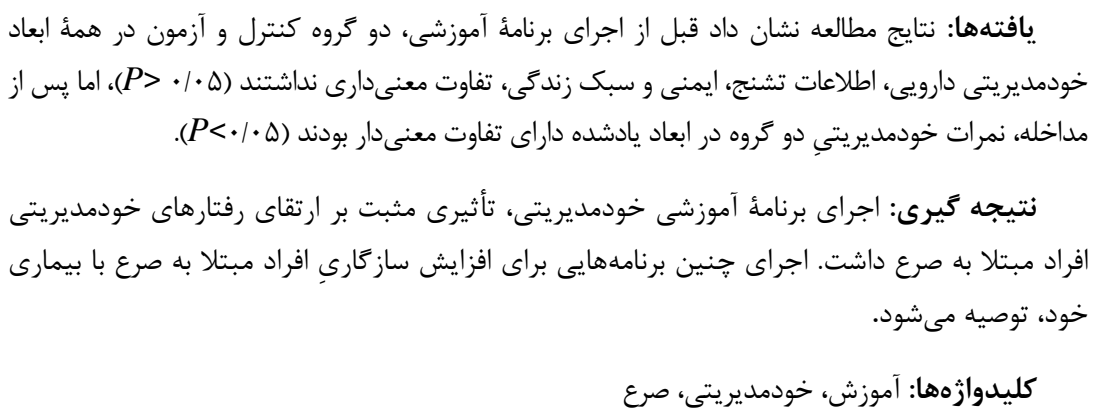 & براى دانلود اين مقاله، كد زير رايد \\
\hline
\end{tabular}

مقدمه

مشكلات روانى اجتماعى همجون افسردگى، اختلالات اضطرابى،

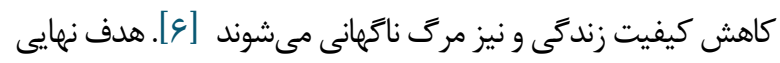

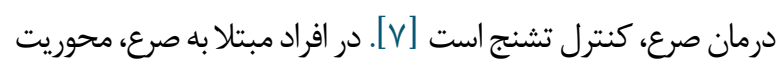

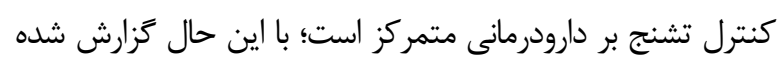

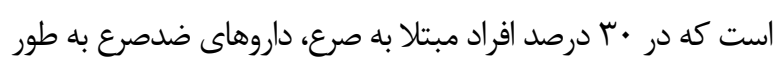

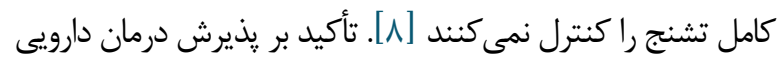

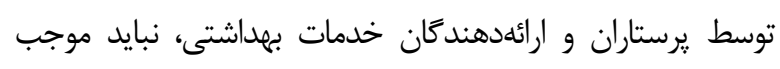

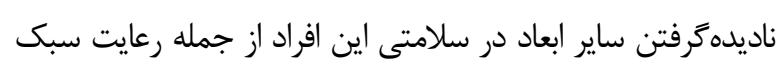

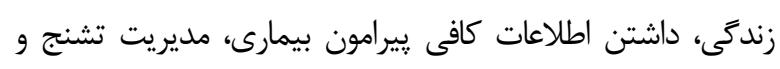

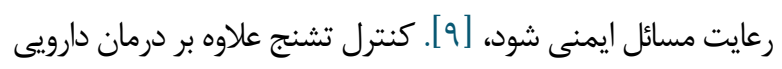

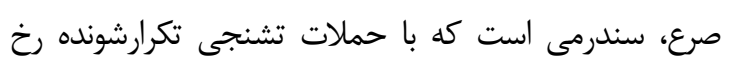

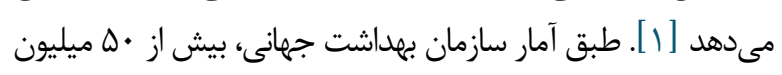

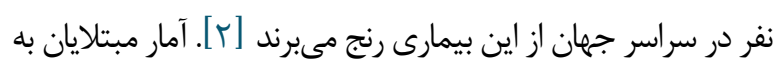

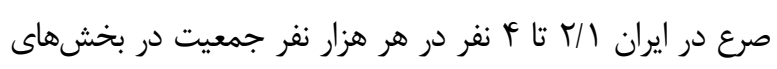
مختلف كشور ززارش شده است [ب].

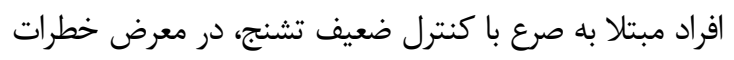

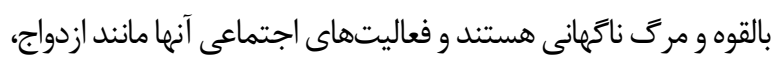

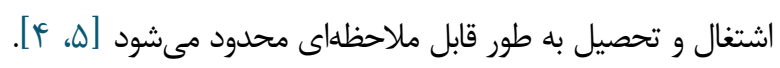
تشنجهاى كنترلنشده نه فقط موجب آسيبهاى جسمان مسمانى ازجمله

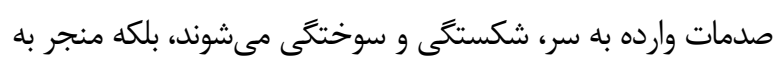


نر كس بحيرائى و همكاران بوب

سال צوץ" اتشكيل دادند. در اين مطالعه، •9 بيمار مبتلا به صرع كه

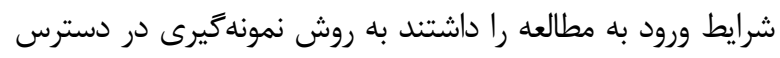

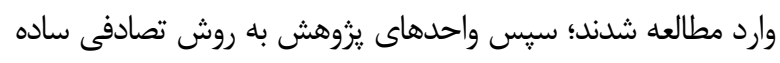

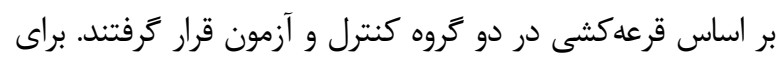

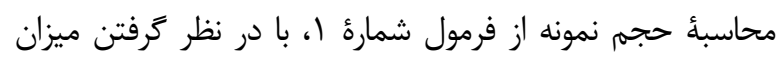
اطمينان هو درصد استفاده شد:

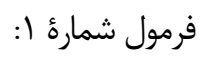

\section{$\left\{\left[2\left(\mathrm{z}_{1-\alpha / 2}+\mathrm{z}_{1-\beta}\right)^{2} \mathrm{~s}^{2}\right] /\left(\mu_{1}-\mu_{2}\right)^{2}\right] \times \mathbf{n}=(\mathbf{1} / \mathbf{1}-\mathbf{f})$}

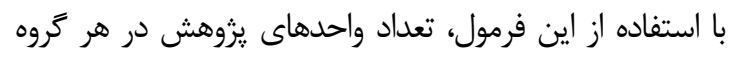
حداقل هأ نفر محاسبه شد. معيارهاى ورود به مطالعه عبارت بودند از: داشتن رضايت آكاهانه

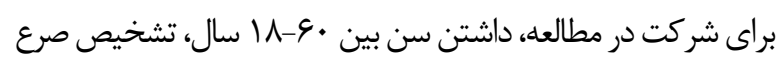

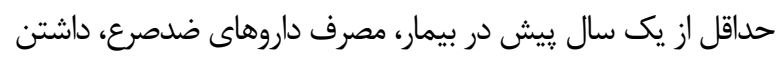

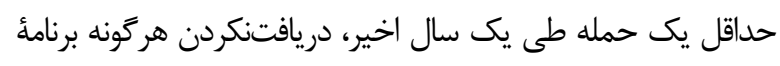

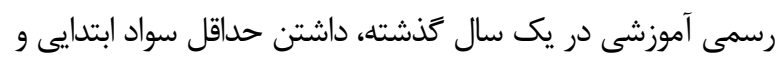

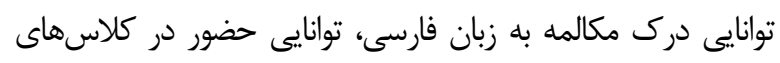

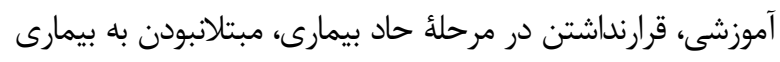

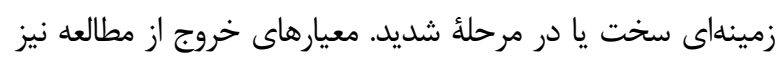

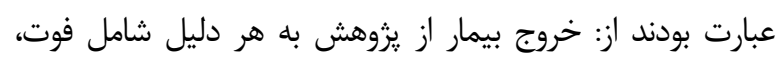

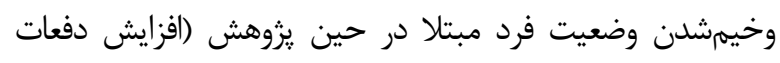

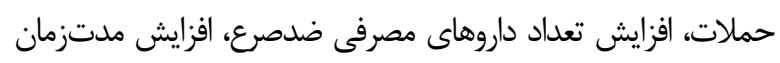

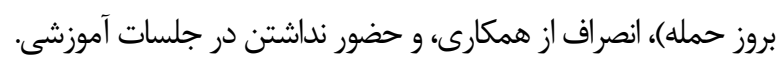
در اين مطالعه، يرسشنامةٔ اطلاعات دموكر افيك (شامل اطلاعات مربوط به سن، جنس، وضعيت تأهل، وضعيت اشتغال، وضعيت اقتصادى، محل سكونت، سطح تحصيلات)، فرم مشخصات بن بيمارى

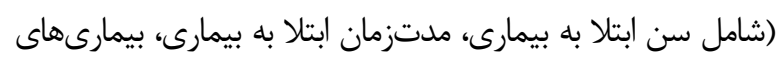

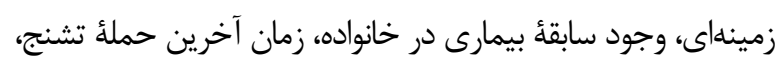

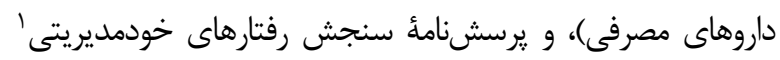

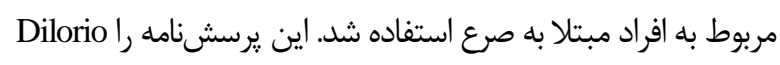

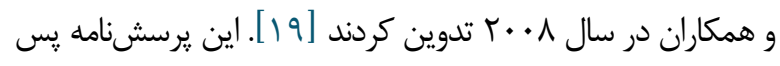

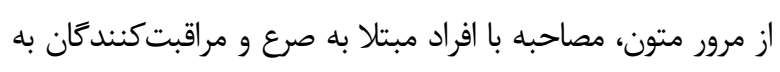

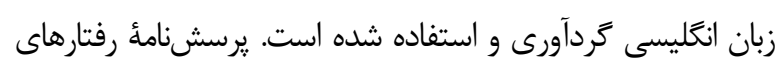

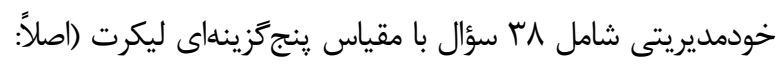

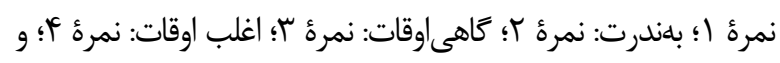

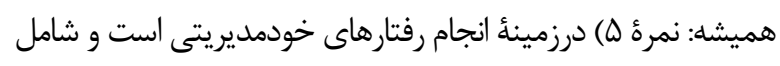
ينج بُعد خودمديريتي دارودرمانى (• سؤال)، اطلاعات (^ سؤال)،
مناسب، به رفتارهاى خودمديريتى وابسته است [ • 1] خودمديريتى

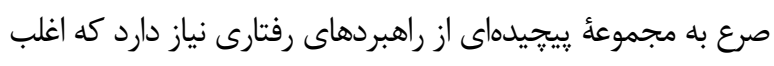

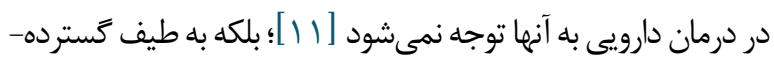

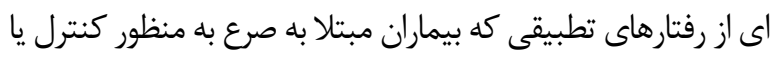

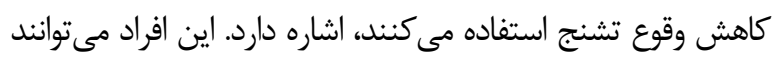

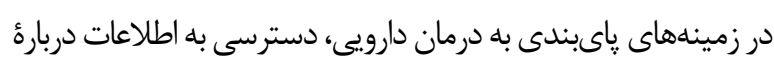

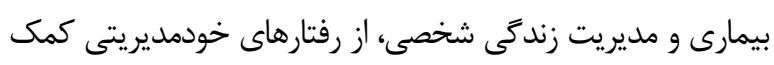

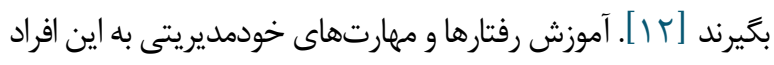

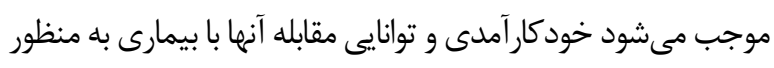

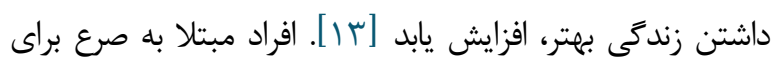

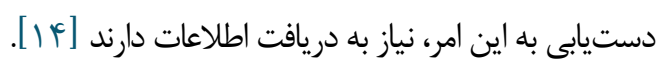

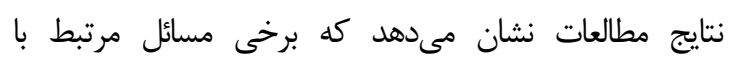

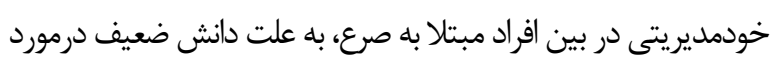

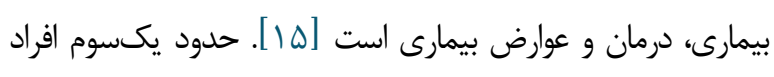

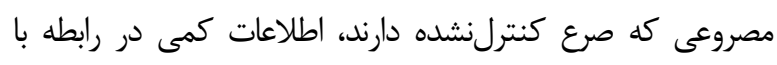

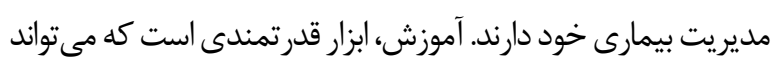

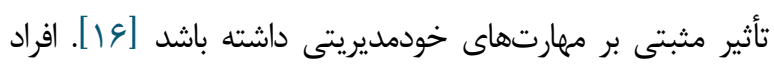

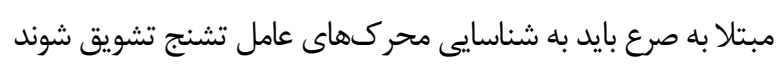

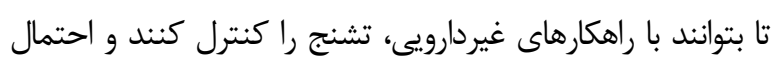

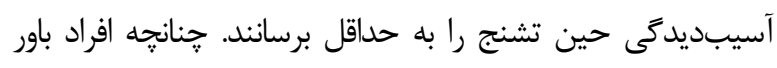

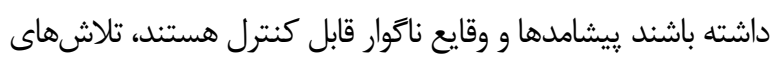

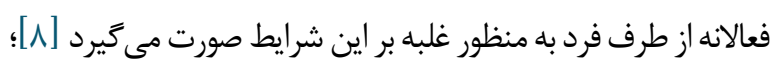

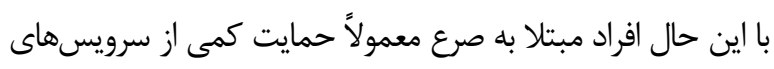

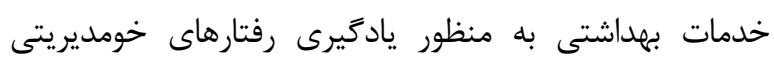

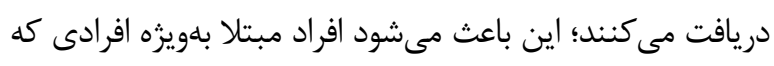

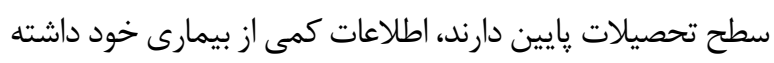

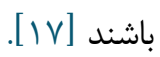

درزمينٔ بيمارى هاى مزمن از جمله صرع، يرستاران نقش كليدى

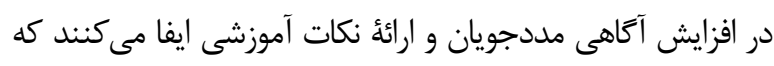

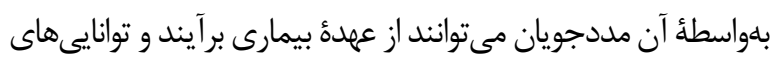

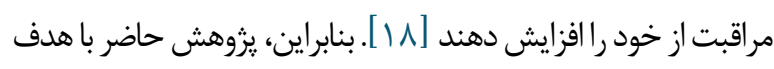

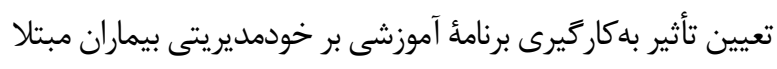
به صرع انجام شد. روش كار

اين يزوهش به روش نيملتجربى انجام شد. جامعأ آمارى آن را

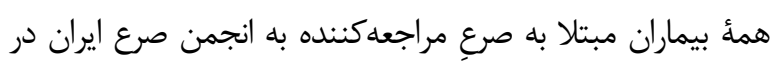

${ }^{1}$ Epilepsy Self-management Scale 
ايمنى در اتاق نشيمن، ايمنى در اتاق كار، ايمنى در حياط) بركزار شد. محتواى جلسة سوم نيز آموزش خودمديريتى تشنج (شامل

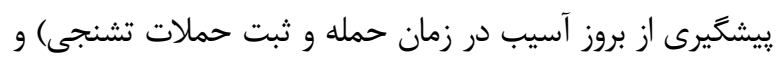

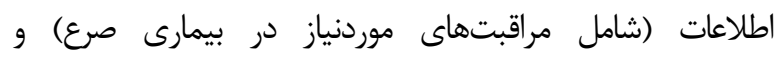
خودمديريتى سبك زندگى (مديريت استرس، خواب، تغذيه و ورزش)

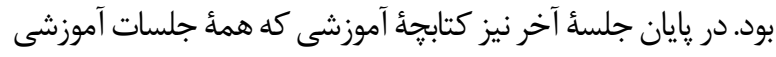

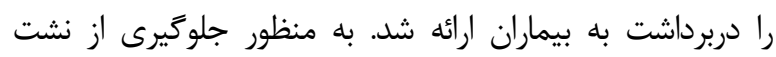

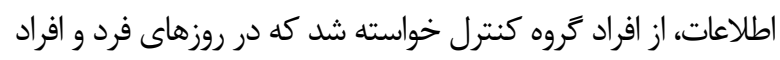

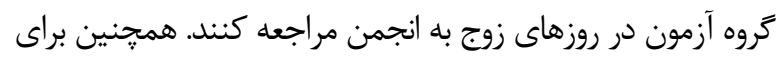

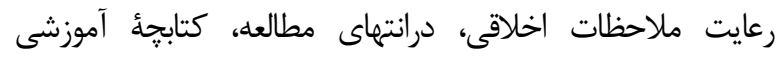

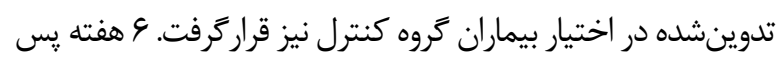
از آخرين جلسئ آموزشى، بار ديخر بيماران هر دو كروه، يرسشنامنامها و فرم مشخصات بيمارى را تكميل كردند.

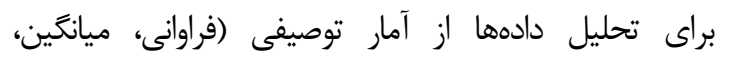

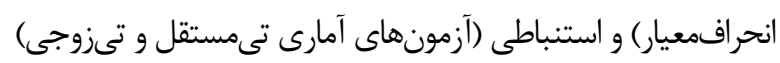

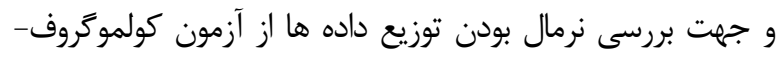

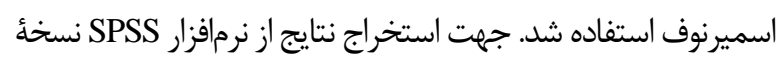
(SPSS Inc., Chicago, Ill., USA) Tr معنادارى كمتر از ه • • در نظر ترفته شد.

\section{يافته ها}

نتايج حاصل از يزوهش نشان داد بين دو كروه كنترل و آزمون،

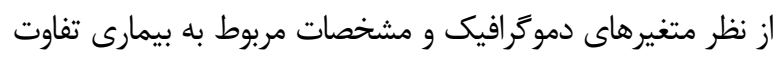

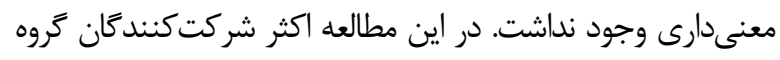

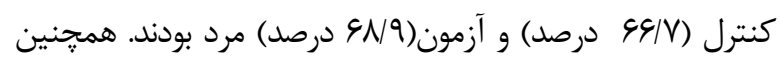

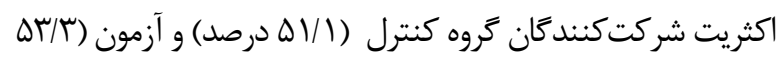

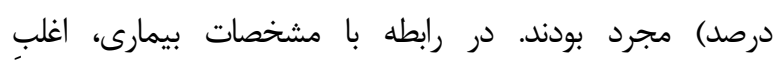

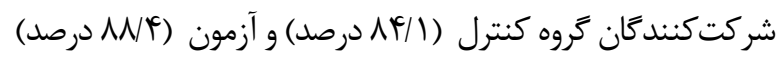
رزيم جنددارويى مصرف مىكردند

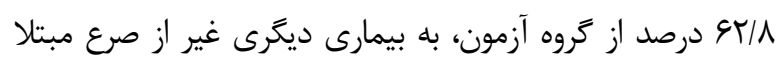

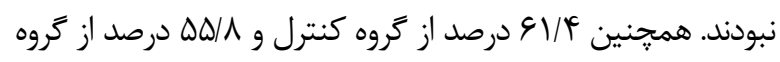

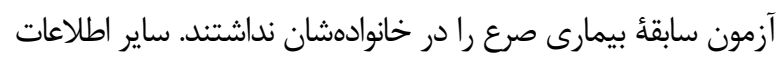

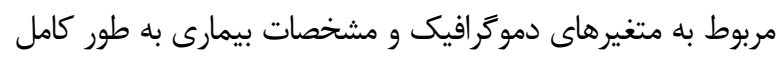
در جدول شماره ا ذكر شده است.

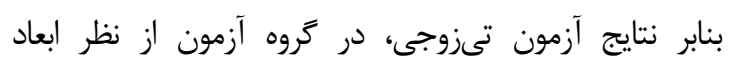

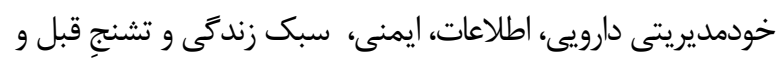

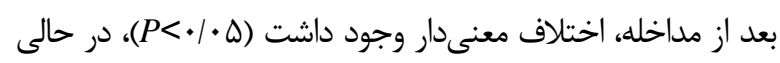

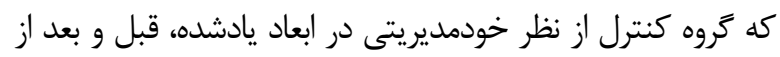

ايمنى (1 سؤال)، تشنج (9 سؤال) و سبك زندگى (ع سؤال) مىشود.

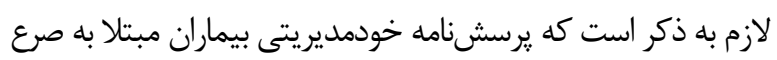

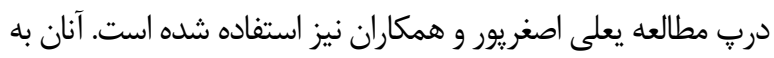

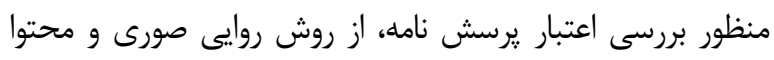

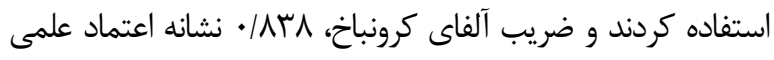

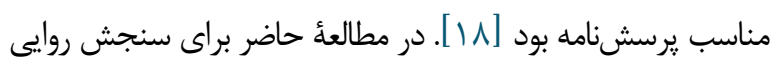

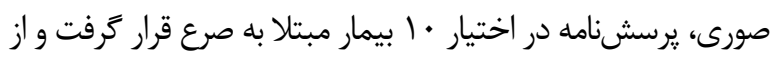

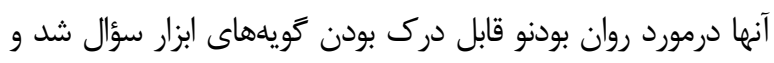

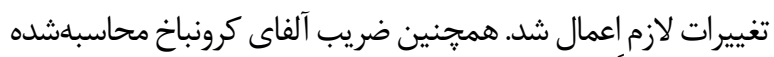

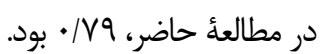
جهت انجام يزوهش،يس از كسب معرفى نامئ كتبى و اخذ مجوز

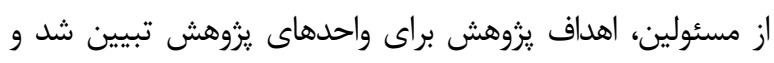

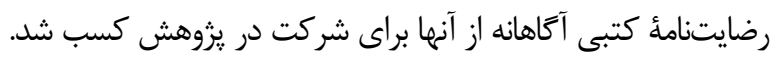

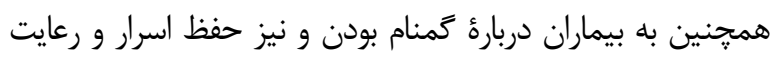

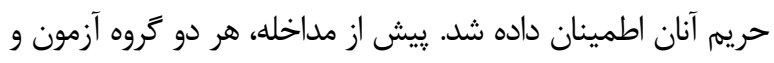

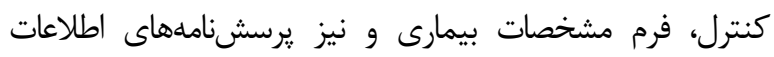

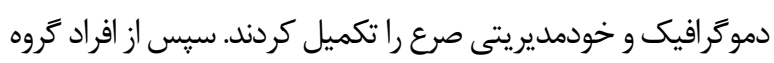

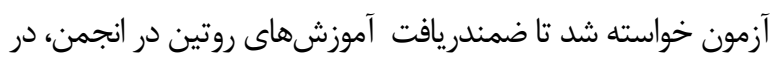

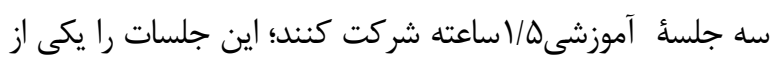

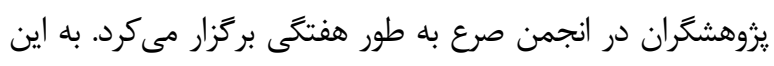

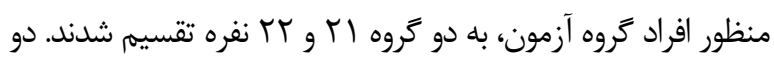

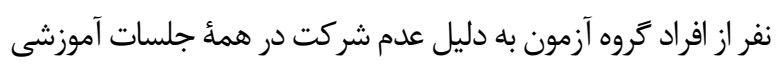
و يك نفر از گروه كنترل، به دليل انصراف از همكارى از از مطالعه خارج

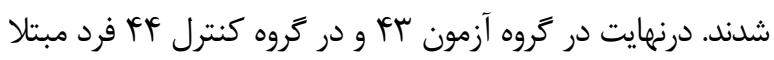

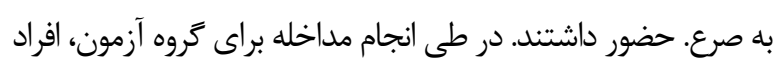

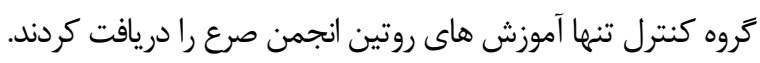

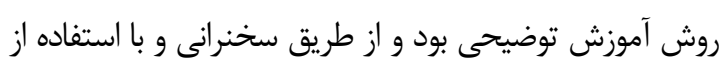

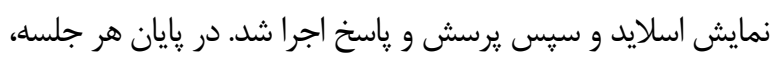

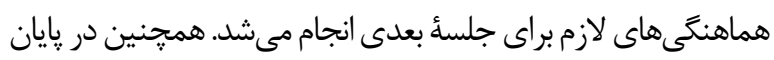

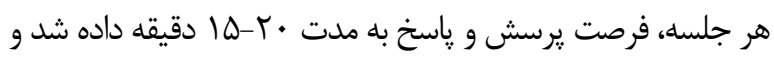

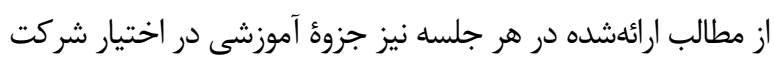

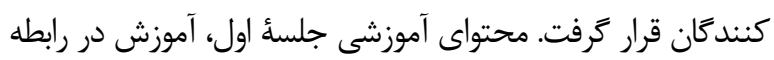

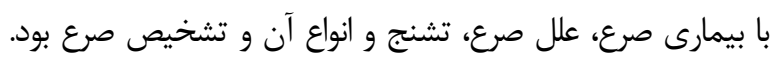

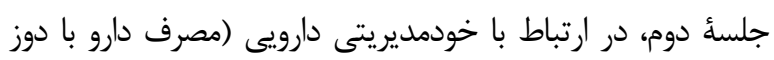

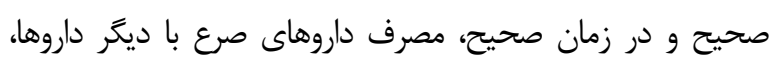

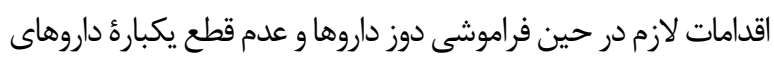

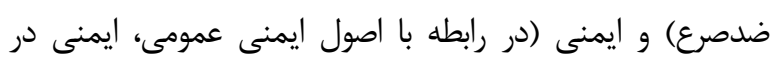
آشيزخانه، ايمنى در حمام، ايمنى هنكام خواب، ايمنى در راهيل إيلهها، 


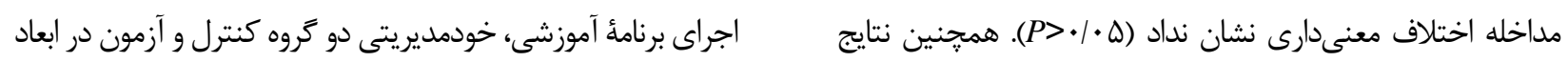

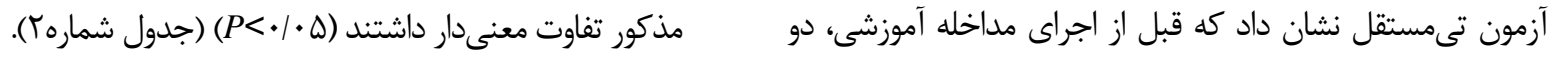

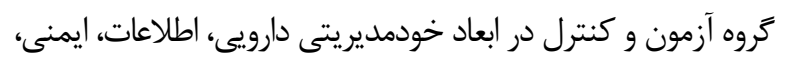

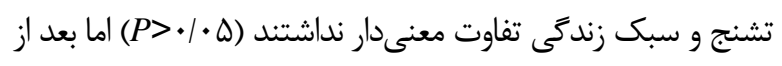

جدول شماره ا: مقايسهُ متغير هاى دموكرافيك افراد مبتلا به صرع به تفكيك كروه آزمون و كنترل

\begin{tabular}{|c|c|c|c|c|c|c|}
\hline \multirow{2}{*}{ نتيجه آزمون } & \multicolumn{2}{|c|}{ تروه كنترل } & \multicolumn{2}{|c|}{ تروه آزمون } & \multirow{2}{*}{ تروه } & \multirow{2}{*}{ متغير } \\
\hline & درصد & 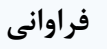 & درصد & فراوانى & & \\
\hline \multirow{3}{*}{$\begin{array}{c}\mathrm{t}=\cdot / \kappa \uparrow \varphi \\
\mathrm{df}=\wedge \wedge \\
P=\cdot / \vee \uparrow \Delta\end{array}$} & rN/G & IV & $F F / T$ & 19 & كمتر از • • سال & \multirow{3}{*}{ سن } \\
\hline & $r F / l$ & 10 & $r \cdot / r$ & r & 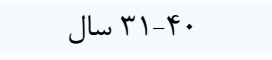 & \\
\hline & $r V / r$ & ir & $r \Delta / \varphi$ & 11 & بالاى • f سال & \\
\hline \multirow{2}{*}{$P=\cdot / \Lambda T$} & $9 N / T$ & $r \cdot$ & $9 \Delta / 1$ & $r \wedge$ & مرد & \multirow{2}{*}{ جنسيت } \\
\hline & rI/ & 14 & $r F / q$ & 10 & 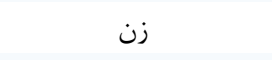 & \\
\hline \multirow{2}{*}{$P=1$} & $\Delta T / T$ & r & $\Delta r / \Delta$ & rr & مجرد & \multirow{2}{*}{ 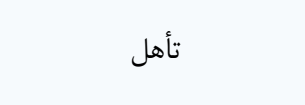 } \\
\hline & $q V / V$ & rI & $\varphi \& / 0$ & $r \cdot$ & متأهل & \\
\hline \multirow{4}{*}{$P=\cdot / r \vee q$} & $1 N / T$ & $\wedge$ & $\cdot / V$ & $r$ & ابتدايى & \multirow{4}{*}{ تحصيلات } \\
\hline & $r q / \Delta$ & ir & rQ/Q & 11 & متوسطه & \\
\hline & $r \cdot 10$ & 9 & $T \Delta / 9$ & 11 & دييلم & \\
\hline & ri/A & If & $41 / 9$ & 11 & دانشخاهى & \\
\hline \multirow{2}{*}{$P=\cdot / r V F$} & $\Delta T / r$ & r & 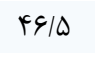 & $r \cdot$ & شاغل & \multirow{2}{*}{ وضعيت اشتغال } \\
\hline & $F V / V$ & rI & $\Delta r / \Delta$ & r & بيكار & \\
\hline \multirow{3}{*}{$P=\cdot / 9 \wedge \vee$} & $1 r / 9$ & 9 & If & 9 & خوب & \multirow{3}{*}{ وضعيت اقتصادى } \\
\hline & rI/A & If & $r \cdot / r$ & ir & 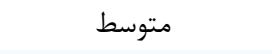 & \\
\hline & $\Delta F / \Delta$ & TF & $\Delta \Delta / \wedge$ & Tr & ضعيف & \\
\hline \multirow{3}{*}{$P=\cdot / 9$} & $9 \Delta / 9$ & rq & V/VG & س & يك ماه كذشته & \multirow{3}{*}{ آخرين تشنج } \\
\hline & $r V / r$ & ir & $11 / 9$ & $\wedge$ & بين ا تا و ماه كذشته & \\
\hline & $9 / 1$ & $r$ & $r / v$ & r & بين 9 تا זا ماه كذشته & \\
\hline \multirow{2}{*}{$\begin{array}{l}\mathrm{t}=\cdot / 1 \wedge \mathrm{r} \\
\mathrm{df}=\wedge \Delta\end{array}$} & $r q / 9$ & ir & $r \Delta / \varepsilon$ & 11 & كمتر از & \multirow{3}{*}{ سن ابتلا (سال) } \\
\hline & rN/G & iv & $F \& / T$ & 19 & G-r. & \\
\hline$P=\cdot \mid \wedge \Delta \varphi$ & $M I / \Lambda$ & If & $r \cdot / r$ & M & بالاى · r & \\
\hline \multirow{2}{*}{$\begin{array}{l}\mathrm{t}=\cdot / \cdot \wedge \cdot \\
\mathrm{df}=\wedge \Delta \\
P=\cdot / 9 \mu \mathrm{V}\end{array}$} & $\Psi V / V$ & rI & $F F / T$ & 19 & كمتر از • r ماه & \multirow{3}{*}{ مدت ابتلا (ماه) } \\
\hline & $r q / 9$ & Ir & $r q / \Delta$ & iv & 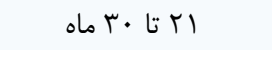 & \\
\hline $\begin{array}{c}P=\cdot / 9 \mathrm{~V} \\
\cdot / 9 \mathrm{VV}\end{array}$ & $T r / V$ & 1. & $18 / \pi$ & v & بيش از • ب ماه & \\
\hline \multirow[t]{2}{*}{$P=\cdot / V \Delta V$} & $10 / 9$ & $\checkmark$ & $11 / 9$ & $\Delta$ & 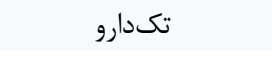 & \multirow{2}{*}{ داروهاى مصرفى } \\
\hline & $\Lambda F / 1$ & rv & $M N / F$ & rی & 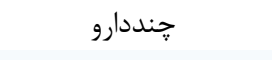 & \\
\hline \multirow{2}{*}{$P=\cdot \mid \varphi \Delta \varphi$} & $\mathrm{r} I / \Lambda$ & If & $r V / r$ & 19 & 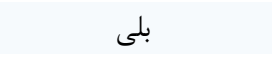 & \multirow{2}{*}{ ابتلا به بيمارى ديخر } \\
\hline & $9 N / T$ & $r \cdot$ & GY/A & TV & خير & \\
\hline$P=\cdot / r \Delta \varphi$ & $V \mathrm{~V} / \mathrm{r}$ & mF & $\Lambda N / \uparrow^{q}$ & ґ^ & 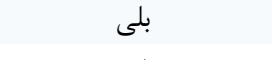 & برخورداري از بيمه درماني \\
\hline a dict & $T r / V$ & $1 \cdot$ & $11 / 9$ & $\Delta$ & 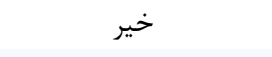 & \\
\hline$P=.1994$ & rN/G & IV & $F \& / T$ & 19 & بلى بلى & سابقة صرع در خانواده \\
\hline & $91 / 4$ & TV & $\Delta \Delta / \wedge$ & TF & خير & \\
\hline$P=\cdot 194$ & $r F / l$ & 10 & rq/q & IV & 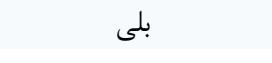 & در بافت د نامئ آمهز شـ ديخ \\
\hline & $9 D / 9$ & rq & $9 \cdot 10$ & re & خير & 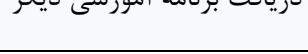 \\
\hline
\end{tabular}


جدول شماره rا: مقايسه بعد خودمديريتى ايمنى، تشنج و اطلاعات قبل و بعد از مداخللُ آموزشى در گروه آزمون و كنترل

\begin{tabular}{|c|c|c|c|c|c|c|}
\hline \multirow{2}{*}{ آزمون تىمستقل } & \multicolumn{2}{|c|}{ كنترل } & \multicolumn{2}{|c|}{ آزمون } & \multirow{2}{*}{ تروه } & \multirow[b]{2}{*}{ متغير } \\
\hline & انحر اف معيار & ميانغين & انحر افمعيار & ميانغين & & \\
\hline $\begin{array}{c}\mathrm{t}=1 / \Delta f q \\
\mathrm{df}=\wedge \Delta \\
P=\cdot / / r \Delta\end{array}$ & F/QT & $T r / V$. & F/r & $r F / 19$ & پيشآزمون & خودمديريتى \\
\hline $\begin{array}{c}\mathrm{t}=r / \mathrm{r} \wedge \\
\mathrm{df}=\wedge \Delta \\
P<\cdot / \cdot \cdot 1\end{array}$ & $F / \Delta \varepsilon$ & $r Y|q|$ & $r / T \cdot$ & $r \Delta / r q$ & يֶ آزمون & ايمنى \\
\hline $\begin{array}{c}\mathrm{t}=\cdot / / \wedge \Delta \\
\mathrm{df}=\wedge \Delta \\
P=\cdot / \vee १ ৭\end{array}$ & $\Delta / \cdot 9$ & $19 / 1 F$ & $\Delta / r \Delta$ & $19 / 09$ & يَيش آزمون & $\therefore$ " \\
\hline $\begin{array}{c}\mathrm{t}=\mathrm{V} / \mathrm{lr} \mathrm{\varepsilon} \\
\mathrm{df}=\wedge \mathrm{d} \\
P<\cdot / \cdot \cdot 1\end{array}$ & $\Delta /|T|$ & $19 / 91$ & $T / V F$ & $T \&|r|$ & يس آزمون & ودمديريتى تشنج \\
\hline $\begin{array}{c}\mathrm{t}=\cdot / 1 \mathrm{~F} \varphi \\
\mathrm{df}=\wedge \Delta \\
P=\cdot / \Lambda \wedge \uparrow\end{array}$ & $\varphi / \wedge \varphi$ & $19 / 11$ & $\Delta / r \Delta$ & $11 / 90$ & يِيش آزمون & خودمديريتى \\
\hline $\begin{array}{c}\mathrm{t}=\mid r / r \cdot \mathrm{f} \\
\mathrm{df}=\wedge \Delta \\
P<\cdot / \cdot \cdot 1\end{array}$ & $\Delta / \cdot F$ & IN/NF & $r / V I$ & $T I / Q I$ & يس آزمون & اطلاعات \\
\hline $\begin{array}{c}\mathrm{t}=1 / \Delta \& q \\
\mathrm{df}=\wedge \Delta \\
\mathrm{p}-\mathrm{value}=\cdot / / \mathrm{r} \Delta\end{array}$ & T/Tr & $r r / \Lambda q$ & $F / \Delta \Lambda$ & r r/gr & يِش آزمون & خودمديريتى \\
\hline $\begin{array}{c}\mathrm{t}=r / \mathrm{V} r \wedge \\
\mathrm{df}=\wedge \Delta \\
\mathrm{p} \text {-value }<0 / 001\end{array}$ & $F / M F$ & TF & T/KT & r\&/\&D & يس آزمون & دارويى \\
\hline $\begin{array}{l}\mathrm{t}=\cdot / \mathrm{FFr} \\
\mathrm{df}=\wedge \Delta\end{array}$ & $\Delta / r T$ & $19 / V \Delta$ & $\Delta / f \Delta$ & IV/TG & پيش آزمون & \\
\hline $\mathrm{p}$-value $=\cdot / 49$. & & & & & & خودمديريتى سبك \\
\hline $\begin{array}{c}\mathrm{t}=\wedge / \mathrm{\Delta} \boldsymbol{q} \\
\mathrm{df}=\wedge \Delta \\
\mathrm{p} \text {-value }<0 / 001\end{array}$ & $\Delta / \cdot \Lambda$ & IV/TK & r/9r & $r \Delta / 19$ & يس آزمون & زندگى \\
\hline
\end{tabular}

\section{بحث}

مطالعُ McLaughlin و بأ عنوان المطالعُ كارآزمايى مبتنى بر برنامة درمانى شناختىرفتارى براى

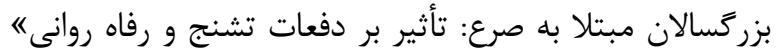

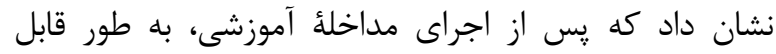

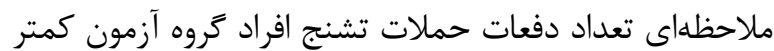

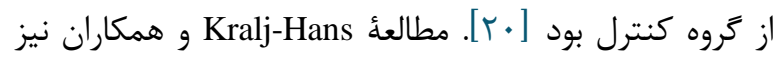
نشان داد كه برنامئ آموزش خودمديريتى در بهبود كيفيت

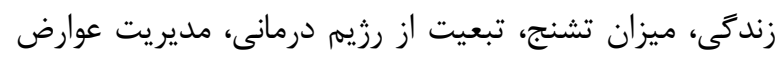

اين مطالعه با هدف تعيين تأثير بهكارگيرى برنامهٔ آموزشى

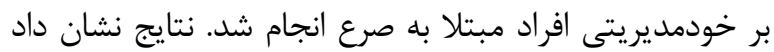
ميانگين نمره خودمديريتى كنترل تشنج، ايمنى، اطلاعات، سبك زندگى و دارويى در گروه آزمون، قبل و بعد از مداخله

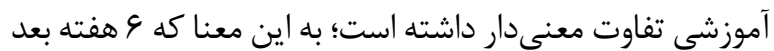
از انجام مداخلئ آموزشى، خودمديريريتى گروه آزمون در ابعاد ياد شده افزايش يافت، درحالى كه اين تفاوت در گروه كنترل قابل توجه نبود. 
نركس بحيرائى و همكاران VVV

مشكلات آموزشى و يادَيرى در بيماران مبتلا به صرع، استفاده از

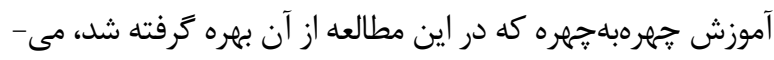

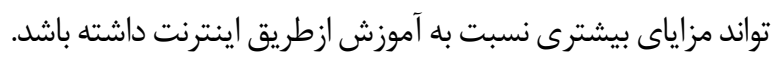
در مطالعه مeethalakshemi و همكاران، سلماه بعد از انجام مداخله آموزشى، ميانگين نمرءٔ خودمديريتى افراد كروه مداخله

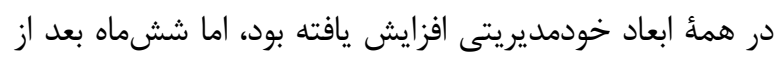

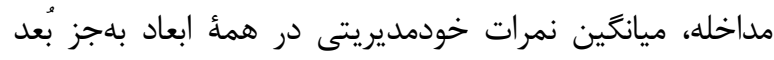

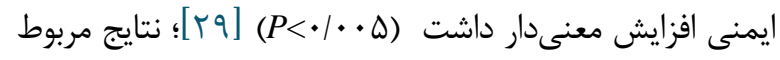

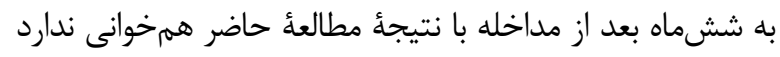

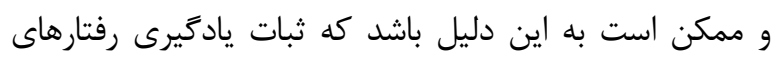

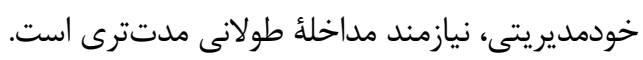

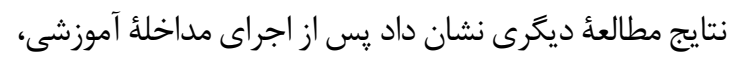

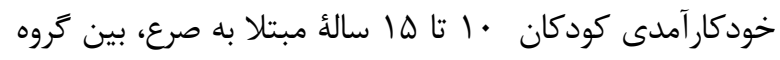

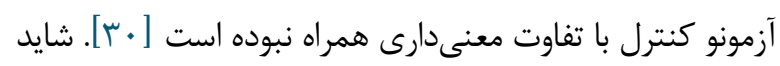

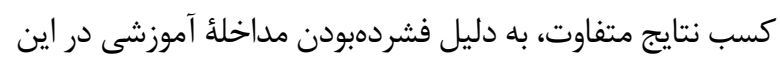

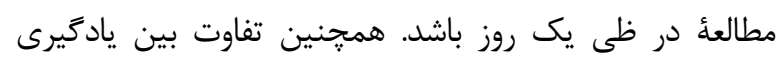

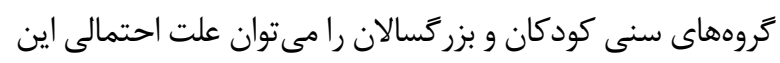

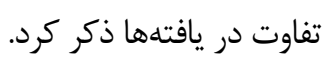

\section{نتيجة نهايى}

نتايج مطالعه نشان داد آموزش، تأثيرى مثبت بر رفتارهاى

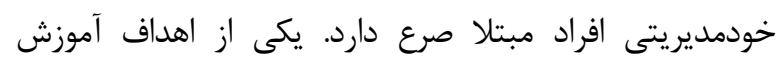

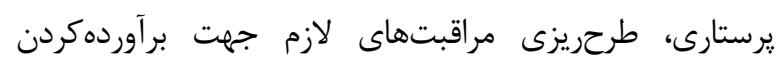

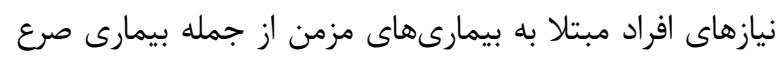

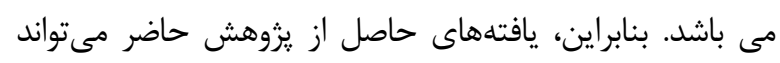

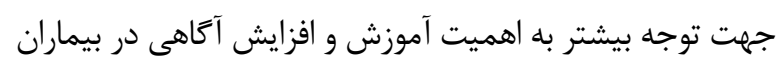

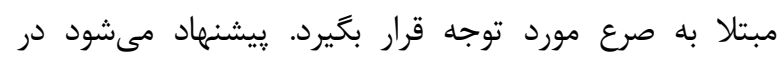

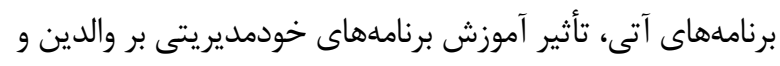

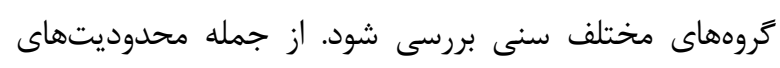

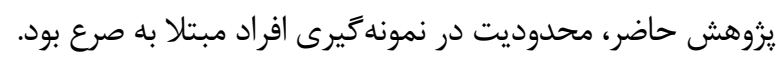

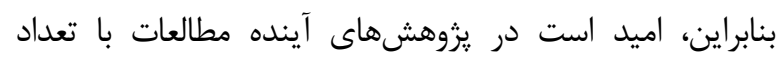
نمونههاى بيشتر انجام گيرد.

\section{سياسگزارى}

يزوهش حاضر بخشى از پاياننامٔ كارشناسى ارشد با

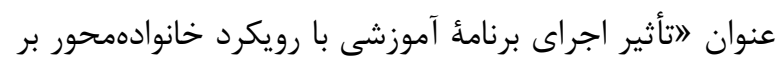

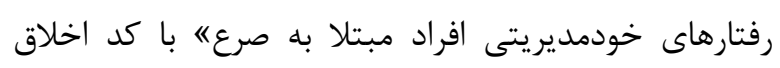

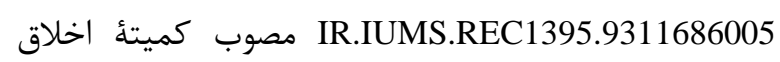

دارويى و بهببود خودكار آمدى در افراد مبتلا به صرع تأثير مثبت

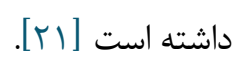
بر اساس مطالعه Tang، آموزش دارويى و پِيخيرى تلفنى،

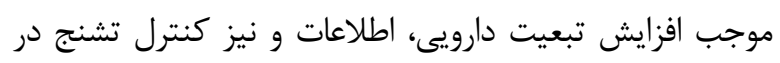

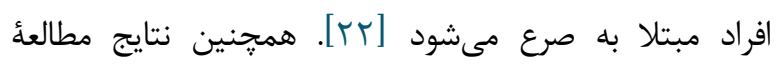
Laybourne مبتلا به صرع باعث افزايش دانش و اعتمادبهنفس دان در مداد مدئل

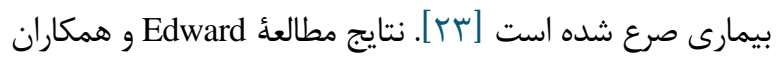

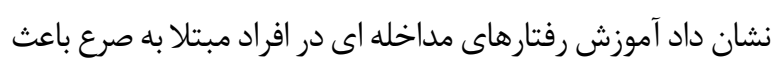

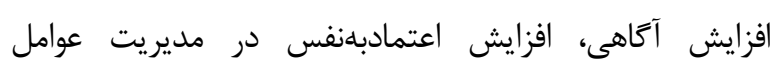

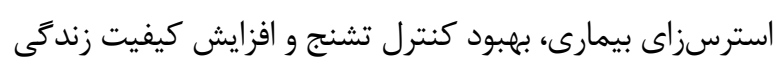

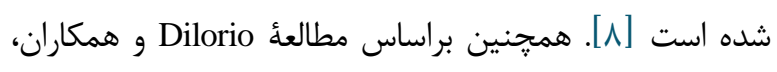

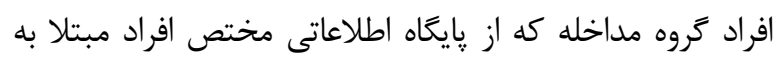

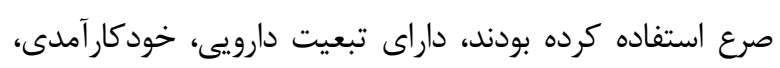

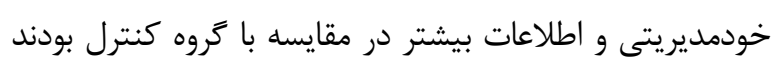

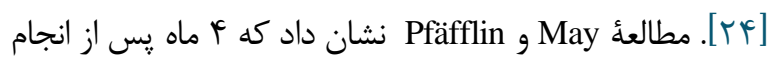

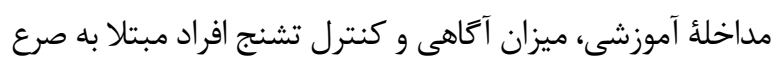

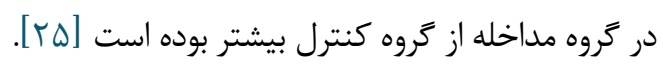
Aliasgharpour

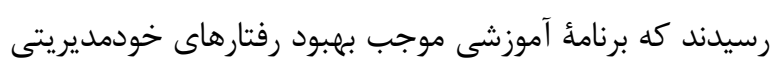

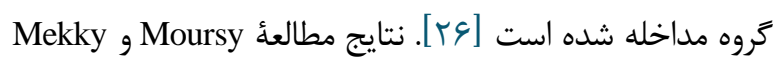

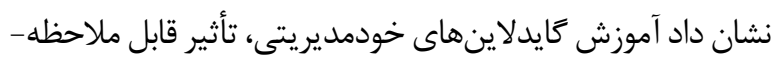

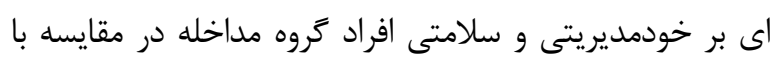

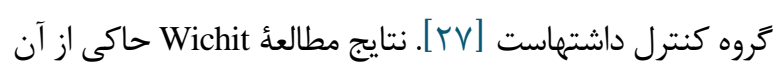

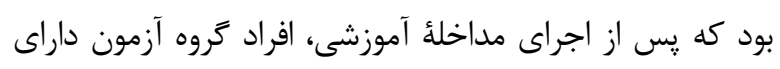

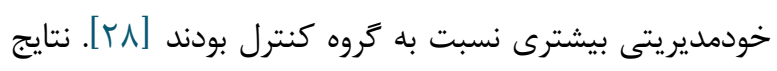
همكى اين مطالعات با يافتهاى مطالعأ حاضر همخدوانى دارند.

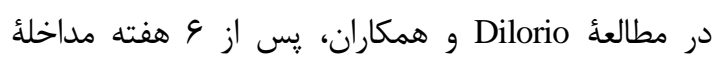

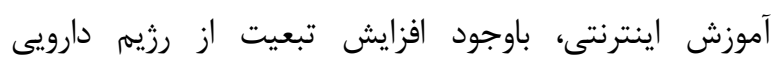

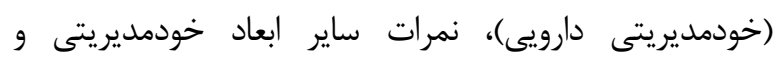

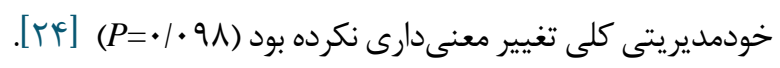

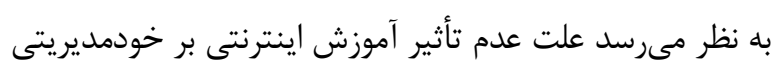

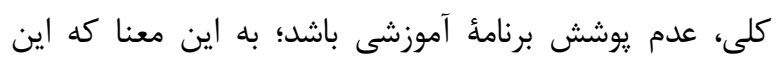

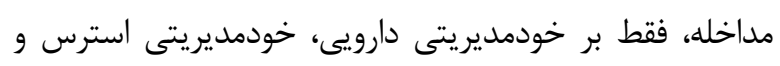

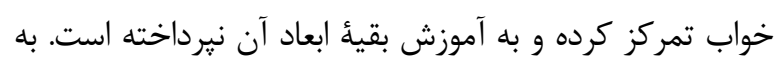

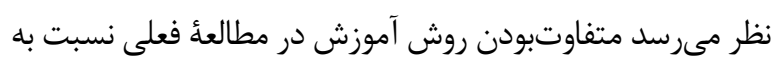

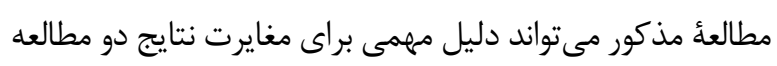

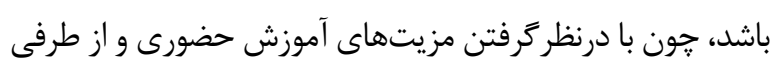




$$
\begin{aligned}
& \text { تعارض در منافع } \\
& \text { بين نويسند كان هيجگَنه تعارضى در منافع وجود ندارد }
\end{aligned}
$$

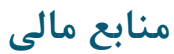

$$
\begin{aligned}
& \text { منابع مالى اين مطالعه توسط نويسندكًان تامين شده است. }
\end{aligned}
$$

$$
\begin{aligned}
& \text { دانشگاه علوميزشكى ايران و به شماره } 19812 \text { ثبتشده در مركز } \\
& \text { كارآزمايى بالينى است. بدين وسيله از همكاران محترم دانشعاه } \\
& \text { علوميزشكى ايران، انجمن صرع ايران و معاونت يزوهشى و وائى }
\end{aligned}
$$

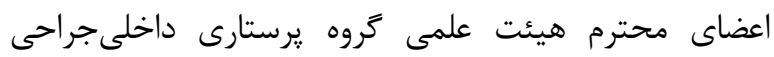

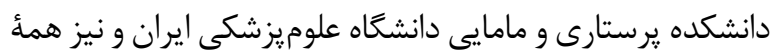

$$
\begin{aligned}
& \text { بيمارانى كه در اين مطالعه شركت كردند تشكر مى كنيم. }
\end{aligned}
$$

\section{References}

1. Smeltzer SC, Bare BG, Hinkle JL, Cheever KH. Brunner \& Suddarht's of textbook of Medical- Surgical Nursing. 12th edition .Philadelphia: Lippincott Williams \& Wilkins; 2010.

2. Binagwaho A, Cohen L, Galea S, Grover A, Jabbour S, Jha V, Kickbusch L, Kieny MP, Land MA, Majdzadeh R, Nuwayhid I, Saxena S, Seng CK, Sissay L, Woodward A. Epilepsy.Available at:URL:http: //www.who.int/.Accessed :March 7,2012

3. Masoud SA, Kochaki E. Surveying the family attitude of a patients with epilepsy hospitalized in Shahid Beheshti Hospital in Kashan, 1378-79. Feyz. 2004; 8(1):79-86.

4. Oh A, Thurman DJ, Kim H. Comorbidities and risk factors associated with newly diagnosed epilepsy in the US pediatric population. Epilepsy \& Behavior. 2017; 75:230-60 [DOI:10.1016/j.yebeh.2017.07.040] [PMID]

5. Perzynski AT, Ramsey RK, Colón-Zimmermann K, Cage J, Welter E, Sajatovic M. Barriers and facilitators to epilepsy self-management for patients with physical and psychological co-morbidity. Chronic Illness. 2017; 13(3):188-203. [DOI:10.1177/1742395316674540] [PMID] [PMCID]

6. Ferrari CMM, de Sousa RMC, Castro LH. Factors associated with treatment non-adherence in patients with epilepsy in Brazil. Seizure. 2013; 22 (5):384-9. [DOI:10.1016/j.seizure.2013.02.006] [PMID]

7. Murray CJ, Vos T, Lozano R, Naghavi M, Flaxman $\mathrm{AD}$, Michaud C, et al. Disability-adjusted life years (DALYs) for 291 diseases and injuriesin 21 regions, 1990-2010: a systematic analysis for the Global Burden of Disease Study 2010. Lancet. 2012; 380(9859):2197. 223. [DOI:10.1016/S0140-6736(12)61689-4]

8. Edward KL, Cook M, Giandinoto JA. An integrative review of the benefits of self-management interventions for adults with epilepsy. Epilepsy \& Behavior. 2015; 45:195-204. [DOI:10.1016/i.yebeh.2015.01.026] [PMID]

9. Ashktorab T, Yadollahi S, Safavi Bayat Z, Zayery F. The correlation between self-management behaviors and drug adherence among people with epilepsy in Iran Epilepsy Association. Scientific Journal of Hamadan Nursing \& Midwifery Faculty. 2013; 21(2):5-15.

10. Ridsdale L, Wojewodka G, Robinson EJ, Noble AJ, Morgan M, Taylor SJ, et al. The effectiveness of a group self-management education course for adults with poorly controlled epilepsy, SMILE (UK): A randomized controlled trial. Epilepsia. 2018; 59(5):1048-61. [DOI:10.1111/epi.14073] [PMID] [PMCID]

11. Smithson WH, Hukins D, Buelow JM, Allgar V, Dickson J. Adherence to medicines and selfmanagement of epilepsy: a community-based study. Epilepsy \& Behavior. 2013; 26(1):109-13. [DOI:10.1016/j.yebeh.2012.10.021] [PMID]

12. Xiao X, Si Y, Mo Q, Liu Y, Li C, Zhao J, et al. Development and validation of the Chinese Version of the Adult Epilepsy Self-Management Scale (C-ESMS) in Western China. Epilepsy Research. 2018; 144:43-8. [DOI:10.1016/j.eplepsyres.2018.04.014] [PMID]

13. Escoffery C, McGee R, Bidwell J, Sims C, ThroppEK, Frazier C, et al. A review of mobile apps for epilepsy self-management. Epilepsy \& Behavior. 2018; 81:62-9. [DOI:10.1016/j.yebeh.2017.12.010] [PMID]

14. Taylor SJ, Pinnock H, Epiphaniou E, Pearce G, Parke HL, Schwappach A, et al. A rapid synthesis of the evidence on interventions supportingself-management for people with long-term conditions. Health Services and Delivery Research. 2014; 2(53):1-580. [DOI:10.3310/hsdr02530] [PMID]

15. Johnson EK, Fraser RT, Miller JW, Temkin N, Barber J, Caylor L, et al. A comparison of epilepsy selfmanagement needs: provider and patient perspectives. Epilepsy \& behavior. 2012; 25(2):150-5. [DOI:10.1016/j.yebeh.2012.07.020] [PMID]

16. Dash D, Sebastian TM, Aggarwal M, Tripathi M. Impact of health education on drug adherence and selfcare in people with epilepsy with low education. Epilepsy \& Behavior. 2015; 44:213-7. [DOI:10.1016/j.yebeh.2014.12.030] [PMID]

17. Noble AJ, Snape DA, Morgan M, Goodacre S, Marson AG, Ridsdale L. PO051 Seizure first aid training for people with epilepsy attending emergency departments, and informal carers.: BMJ Publishing Group Ltd; 2017. [DOI:10.1136/jnnp-2017-ABN.84]

18. Sahebolzamani M, Shakuri A, Aliloo A, Rashidi A. The efficacy of self-care education on knowledge and performance of epileptic patients who referred to selected educational hospitals of Tehran University of Medical Sciences in 2008. The Journal of Urmia University of Medical Sciences. 2010; 20(4):284-9.

19. Dilorio C, Faherty B, Manteuffel B. The development and testing of an instrument to measure self-efficacy in

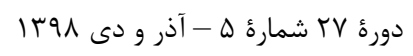

$$
\text { مجله مراقبت يُرستارى و مامايى ابنسينا }
$$


individuals with epilepsy. The Journal of neuroscience nursing: journal of the American Association of Neuroscience Nurses. 1992 Feb;24(1):9-13. [DOI:10.1097/01376517-199202000-00004] [PMID]

20. McLaughlin DP, McFarland K. A randomized trial of a group based cognitive behavior therapy program for older adults with epilepsy: the impact on seizure frequency, depression and psychosocial well-being. Journal of Behavioral Medicine. 2011; 34(3):201-7. [DOI:10.1007/s10865-010-9299-z] [PMID]

21. Kralj-Hans I, Goldstein LH, Noble AJ, Landau S, Magill N, McCrone P, et al. Self-Management education for adults with poorly controlled epilepsy (SMILE (UK)): a randomised controlled trial protocol. BMC neurology. 2014;14 (1):69. [DOI:10.1186/14712377-14-69] [PMID] [PMCID]

22. Tong X, Chen J, Park SP, Wang X, Wang C, Su M, et al. Social support for people with epilepsy in China. Epilepsy \& Behavior. 2016; 64:224-32. [DOI:10.1016/j.yebeh.2016.08.010] [PMID]

23. Laybourne AH, Morgan M, Watkins SH, Lawton R, Ridsdale L, Goldstein LH. Self-management for people with poorly controlled epilepsy: Participants' views of the UK Self-Management in epILEpsy (SMILE) program. Epilepsy \& Behavior. 2015; 52:159-64. [DOI:10.1016/j.yebeh.2015.08.023] [PMID]

24. DiIorio C, Escoffery C, McCarty F, Yeager KA, Henry TR, Koganti A, et al. Evaluation of WebEase: an epilepsy self-management Web site. Health Education $\begin{array}{llll}\text { Research. } & 2008 ; & 24 & \text { (2):185-97. }\end{array}$ [DOI:10.1093/her/cyn012] [PMID]

25. May TW, Pfäfflin M. The efficacy of an educational treatment program for patients with epilepsy (MOSES): results of a controlled, randomized study. Epilepsia. 2002; 43(5):539-49. [DOI:10.1046/j.1528$\underline{1157.2002 .23801 . x}$ ] [PMID]

26. Aliasgharpour M, Nayeri ND, Yadegary MA, Haghani H. Effects of an educational program on selfmanagement in patients with epilepsy. Seizure. 2013; 22(1):48-52. [DOI:10.1016/j.seizure.2012.10.005] [PMID]

27. Moursy AME, Mekky GF. Effect of self management guidelines for adult patients with epilepsy on their health practices. Life Science Journal. 2015; 12(4):7764.

28. Wichit N, Mnatzaganian G, Courtney M, Schulz P, Johnson M. Randomized controlled trial of a familyoriented self-management program to improve selfefficacy, glycemic control and quality of life among Thai individuals with Type 2 diabetes. Diabetes research and clinical practice. 2017; 123:37-48. [DOI:10.1016/j.diabres.2016.11.013] [PMID]

29. Seethalakshmi A, Shankar V. Effectiveness of an epilepsy education programme on the self-management among patients with epilepsy. IOSR Journal of Nursing and Health Science. 2015; 4(5):33-54.

30. Yoo H, Kim HS. Development and evaluation of the Empowering A Self-Efficacy (EASE) program for children with epilepsy. Journal of Korean Academy of
Nursing. 2015 ; [DOI:10.4040/jkan.2015.45.1.54] [PMID]
45(1):54-63. 